Letters to the Editor

\section{Caveats Regarding the Use of Control Charts}

\section{To the Editor:}

A recent article by Dr. Humble ${ }^{1}$ cautioned readers about common problems in applying statistical process control (SPC) charts in health care, perhaps most notably the general importance of selecting an appropriate control chart and correctly calculating the center line and control limits in particular applications. Given the utility and growing use of SPC, the author's caution to avoid such errors is sound advice, and to his list of caveats I also would add the following:

- Creating control charts simply for the sake of creating charts, including widespread computer-generated graphs for almost every possible piece of data, without any real plan on how resultant information will be used. Using SPC requires time and resources, and there is little point in doing any type of analysis unless it will be used to inform or improve.

- Selecting charts based only on what type of data is most readily and easily available, and not on what analysis or statistical power is required. Instead, ideally, explicit questions should dictate analysis methods and chart selection, which in turn should dictate the data that should be collected, not the other way around (retrospectively matching the best chart to preexistent data). I sometimes call this "reverse chart selection" or the "recliner armchair approach." The method requiring the minimum effort often will not yield the best results.

- Not checking or verifying the assumptions of the type of chart being used, such as by simple histograms and autocorrelation tests, thereby increasing the possibility of affecting false alarm rates and the ability to detect process changes.

In addition, a few comments and clarifications regarding specific statements in the above article might be offered, such as with respect to when each type of chart is appropriate. For example, situations in which $p, c$, or $u$ charts should be used are a little confused and in particular are not dependent on the incidence rate as suggested (the author's Table 1). Also, reducing continuous data into simpler dichotomous events results in a loss of statistical power, essentially because the more detailed information in the continuous data is ignored (for example, an increase in radiograph processing time that still is less than some defined cut-point), not due to somehow looser limits. The appropriate chart to use depends on the type of data being examined and the manner of forming these data into subgroups. In summary, $\bar{X}$ and $S$ charts should be used for normal data, $n p$ or $p$ charts for binomial data, $c$ or $u$ charts for Poisson data, and $g$ or $h$ charts for geometric data. Interested readers can see the series by Benneyan ${ }^{2,3}$ or Grant and Leavenworth ${ }^{4}$ for further discussion and clarification.

A second important set of comments concerns the objectives of SPC and when and how best to apply control charts to achieve these. For example, one important purpose is to test or verify whether a known intervention actually produced any demonstrable impact, and control charts would have been an ideal complement (and in fact preferable) to the chisquare tests used in the drug-use evaluation study cited by the author of theophylline-level monitoring in chronic obstructive lung disease patients. Attempts to improve such processes truly are assignable causes only if they can be shown to have an impact on the state of control, favorably or otherwise. Additionally, control charts absolutely should be constructed for processes that are not in states of statistical control. This is precisely one of the primary purposes of SPC, to help bring a process into statistical control by testing for unnatural variability, attempting to stabilize the process, retesting for process consistency, identifying whether interventions improved or worsened the situation, and so on. In fact, how could one even know if a process is or is not in control without using control charts? For similar reasons, it is not necessary to apply runs tests to eliminate out-of-control data before constructing control charts.

Finally, if assumptions of common control charts are not met, sev- eral methods to deal with problems mentioned by the author-such as autocorrelation, limited initial data, and risk adjustment-were described recently ${ }^{2,3}$ in this journal and are the focus of ongoing research. For example, sound methods exist (and frequently are used in other industries) for dealing with autocorrelation for either groups of data or individual data, although these tend to be slightly more complicated and no longer simply plot the raw data as before. For this and other reasons (eg, "armchair SPC"), practitioners should not be encouraged to view control charting merely as superimposing control limits on top of a preexistent run chart.

These clarifications aside, it is encouraging to see an increasing number of articles such as this advocating the importance of using control charts and using them correctly and most effectively. While some industrial applications are by no means simpler and factory workers using SPC with no training is more the exception than the rule, developing a sufficient understanding of control-chart basics and assumptions is important, as in any industry, in order to apply them best to scenarios unique to health care. The best advice is to become both educated and experienced in the use of these tools. To the author's closing remarks that skill is proportional to experience, I therefore would add that skill also is proportional to study, as these should not be confused as synonymous.

\section{REFERENCES}

1. Humble C. Caveats regarding the use of control charts. Infect Control Hosp Epidemiol 1998;19:865-868.

2. Benneyan JC. Statistical quality control methods in infection control and hospital epidemiology, part I: introduction and basic theory. Infect Control Hosp Epidemiol 1998; 19:194-214.

3. Benneyan JC. Statistical quality control methods in infections control and hospital epidemiology, part II: chart use, statistical properties, and research issues. Infect Control Hosp Epidemiol 1998;19:265-277.

4. Grant EL, Leavenworth RS. Statistical Quality Control, 6th ed. New York, NY: McGraw-Hill Book Co; 1988.

James C. Benneyan, PhD Northeastern University Boston, Massachusetts 